\title{
Effective anisotropic elastic constants of bimaterial interphases: comparison between experimental and analytical techniques
}

\author{
CHING-CHANG KO ${ }^{1,3,4}$, D. H. KOHN ${ }^{1,3}$, S. J. HOLLISTER ${ }^{2,3}$ \\ ${ }^{1}$ Department of Biologic and Materials Sciences, School of Dentistry \\ ${ }^{2}$ Orthopaedic Research Laboratories, Department of Surgery \\ ${ }^{3}$ Bioengineering Program University of Michigan Ann Arbor, Michigan 48109-1078 USA
}

The effective elastic constants of a bimaterial composite were experimentally measured with the goal of validating the numerical predications of these constants made by homogenization theory. Secondly, solutions predicted by homogenization theory were compared to predictions made with more standard composite theories. Composite specimens consisting of titanium and epoxy were developed to mimic a porous titanium/tissue interphase. Tensile and shear tests (ASTM D3983) measured the stiffness along the porous coating/epoxy interphase $\left(E_{L}\right)$, across the interphase $\left(E_{T}\right)$ and in shear $\left(G_{\mathrm{LT}}\right)$. No significant differences in moduli were found between the experimental measurements and predictions made with homogenization theory, nor between the experimental measurements and Hashin-Shtrikman estimates. Homogenization theory predicted results usually within $20 \%$ of Hashin-Shtrikman estimates, but typically more than $50 \%$ different from what is predicted by the rule of mixtures. However, homogenization theory allows calculation of anisotropic stiffness estimates and local strains, neither of which is possible using Hashin-Shtrikman estimates. With this experimental validation, the accuracy of homogenization theory for use in implant/tissue interface mechanics applications is confirmed. Since the composite interphase is anisotropic and more compliant in the transverse direction, with stiffness an order of magnitude lower across the interphase, local mechanics, tissue ingrowth and remodeling may be strongly directional dependent.

\section{Introduction}

Porous coated implants have been developed and clinically implemented with the hope of extending the service-life of total joint replacements. This is especially true for younger and more active patients, where some cemented prostheses have 10 -year failure rates over $50 \%$ [1]. In principle, bone tissue can grow into and fill the surface pores, provide a strong implant/tissue interfacial bond through mechanical interlock and improve long-term clinical results.

The implant/tissue interphase, defined here as the composite consisting of the outermost surface of the implant substrate, coating and ingrown tissues, exhibits a unique response to load application. This unique response mechanically distinguishes the interphase from the individual constituents, establishes a specific mechanical environment within the interphase, and may determine the success of the implantation. However, the micromechanical behaviour of the interphase region is not well understood.
For porous metal coated implants, the interphase consists of two major components: the metal coatings and the ingrown tissues - both bony and fibrous tissue. An appropriate diameter and pore size of coating particles, ranging from 50 to $500 \mu \mathrm{m}$, can allow bone to grow into the pores between the particles [2]. The sintering process consolidating powder particles onto the substrate, however, creates notch-like discontinuities on the metal surface, weakening the metal. These notches may serve as loci of stress concentration and initiate crack propagation [3-6].

Ingrown or osseointegrated tissues usually protrude from the surrounding bulk bone and interlock with the coating particles. The loads acting on the implant are expected to be transferred through these bone "spicules". Theoretically, the stress or strain state in these "spicules" may determine whether the mineralized bone is maintained or resorbed after mechanical usage. The remodelling behaviour of the osseointegrated tissues is hypothesized to follow the same principle as bulk bone remodelling - bone mass

${ }^{4}$ Present address: Minnesota Dental Research Center for Biomaterials and Biomechanics, University of Minnesota, Minneapolis, Minnesota 55455 
can be maintained if the bone is subjected to certain mechanical strain ranges [7-9]. Therefore, quantification of localized stresses and strains in the osseointegrated tissue/implant interphase can provide a basis for understanding mechanically mediated bone adaptation and also for improving the design of coated implants.

However, both the complex geometry of the coatings and the small scale of ingrown tissues relative to the entire prosthetic system inhibits micromechanical analysis of the interphase for a whole prosthesis. Finite element analysis (FEA) requires that the element size be smaller than the regions of interest. In the case of a porous coated implant, the local regions of interest are the coating microspheres and bone "spicules" which are on a scale of micrometres. Therefore, FEA of whole implants, on a scale of centimetres, with detailed interphase analysis, on a scale of micrometres is beyond current computational capability.

In general, composite theories are developed to describe macroscopic mechanics by accounting for the effect of constituent materials. Many composite theories have been used to estimate the macroscopic material properties of heterogeneous bimaterial interphases and to provide bounds for the estimated interphase properties of structural materials [10-13]. The macroscopic elastic constants are then used to construct a constitutive law for the interphase in an FEA from which the global or average interfacial mechanical fields can be obtained.

Composite analyses have also been used to simplify the study of bone-implant interphase mechanics [14, 15]. Moyle et al. [14] evaluated the upper and lower bounds on the elastic constants of biomaterial interfaces for different synthetic materials, using both Hill-Paul and Hashin-Shtrikman theories. Their results showed that the Hashin-Shtrikman law predicted narrower bounds than the Hill-Paul method, as is expected. However, no information was presented on the closeness of the bounds to the actual situation. Ducheyne et al. [15], assuming an isotropic interfacial zone, used FEA to investigate the mechanical behaviour of porous-coated implants, in which the elastic modulus of the porous coating was estimated using the Hill-Paul method. These studies provided stress patterns in the prosthesis and surrounding bone away from the interfacial zone. However, only globally isotropic material behaviour was considered. More advanced FEA have evaluated effects of friction and non-linearity at the bone/implant interface [16-18], but a specific implant/tissue interphase was not modelled and local stresses around individual microspheres and adjacent tissue were not determined.

Local single- or multiple-microsphere models have shown that stresses around microspheres are non-uniform and largely dependent upon coating architecture $[6,19,20]$. Additionally, we have demonstrated that an isotropic implant/tissue interphase, derived from the Hill-Paul method, tends to underpredict stresses in metal and overpredict stresses in osseointegrated bone [21].

By applying another composite theory, homogenization theory, we have also showed that the implanttissue interphase composite is macroscopically orthotropic and interphase strains might dictate local failure patterns $[22,23]$. However, to date these results have not been experimentally validated. The purpose of this study was to develop a method of quantifying interphase elasticity accounting for interfacial micro-constituents. Once interphase elasticity is characterized, an appropriate constitutive law may be employed to quantify the interfacial stresses. This study experimentally measured the effective elastic constants $\left(\boldsymbol{C}_{i j}\right)$ of an interphase composite with the goal of validating the numerical predications of these constants. Specifically, the following questions were addressed:

(1) Can homogenization theory predict effective elastic constants of an interphase composite correctly?

(2) Does changing composite constituents affect the resultant effective elastic constants of the interphase?

(3) Is homogenization theory as accurate as other, more standard, composite theories?

\section{Experimental design and methods \\ 2.1. Materials, specimen design and preparation}

Bimaterial composite specimens consisting of titanium and epoxy resins were developed to mimic a composite porous titanium/tissue interphase (Fig. 1). The metal/resin composites included bulk Ti-6 Al-4V, commercially pure titanium (c.p. Ti) microspheres and epoxy. The interphase architecture of the composite was the same as that on the surface of porous coated implants. The titanium powder particles were spherical with a diameter of $1000 \mu \mathrm{m}$. Two isotropic resins were used: epoxy I: $E=0.2 \mathrm{GPa}, v=0.42$; and epoxy II: $E=3 \mathrm{GPa}, v=0.36$ (Table I).

Ti-6Al-4V plates $1 \mathrm{~mm}$ thick were machined into dumb-bell specimens or tensile-lap specimens, which were then split through the $1 \mathrm{~mm}$ thickness along either a vertical or horizontal axis of symmetry. The

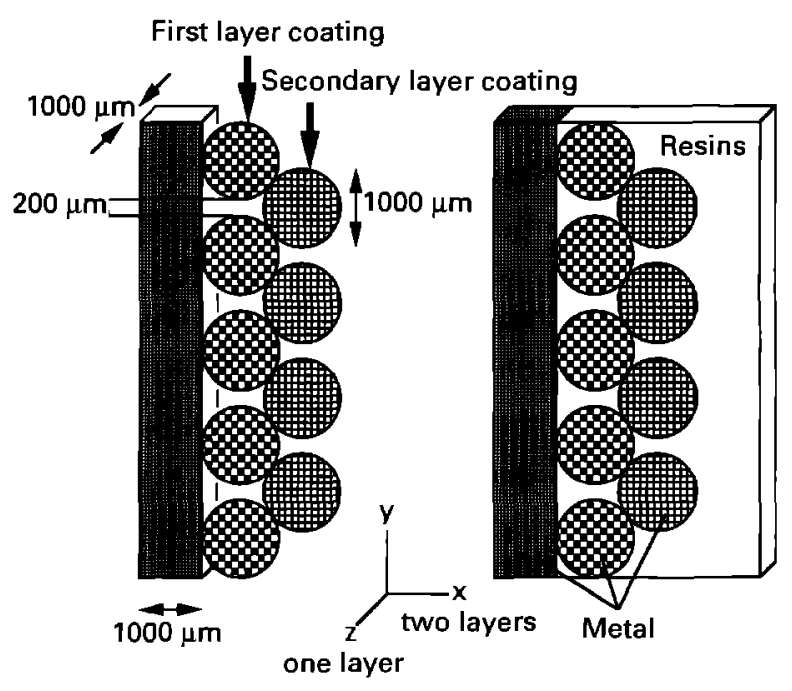

(a)

(b)

Figure I Schematic of composites: (a) Ti-6Al-4V substrate and commercially pure titanium coating; (b) Ti-6Al-4V substrate, commercially pure titanium coating and infiltrated resin. 
TABLE I Elastic constants of constituent materials

\begin{tabular}{llllll}
\hline Material & $\begin{array}{l}\text { Volume } \\
\text { fraction }\end{array}$ & $\begin{array}{l}E \\
(\mathrm{GPa})\end{array}$ & $v$ & $\begin{array}{l}G \\
(\mathrm{GPa})\end{array}$ & $\begin{array}{l}K \\
(\mathrm{GPa})\end{array}$ \\
\hline Titanium & 0.57 & 110 & 0.30 & 42.3 & 91.7 \\
Epoxy I & 0.43 & 0.2 & 0.42 & 0.07 & 0.42 \\
Epoxy II & 0.43 & 3.0 & 0.36 & 1.10 & 3.57 \\
\hline
\end{tabular}

composite was $1 \mathrm{~mm}$ thick to meet the criteria for the plane stress analytical model. Commercially pure titanium powder particles were sintered onto the $1 \mathrm{~mm}$ thick cross-section of the bulk Ti-6Al-4V alloy substrates under high temperatures $\left(\sim 1100^{\circ} \mathrm{C}\right)$. The sintering treatment for bonding the microspheres and substrate produced an $(\alpha+\beta)$ lamellar microstructure and was the same procedure used for coating commercial implants. One layer of coating particles was sintered through the thickness and two layers were sintered across the interphase (Fig. 1). Each microsphere in the first layer across the interphase was sintered to the substrate. Each microsphere in the second layer contacted two microspheres from the first layer.

To make the complete composite specimens, negative moulds were fabricated using silicone rubber impression materials used for denture impressions. By pouring the epoxy resin into the mould, the metal pores were filled with resin. The final titanium/resin composites had a volume fraction of $57 \%$ titanium/ $43 \%$ resin. Finally, the specimens were polished to 600 grit to reduce any surface defects.

\subsection{Testing methods}

The composite interphase was assumed to be orthotropic and have four independent elastic constants for two-dimensional stress-strain relations: the elastic moduli in the longitudinal and transverse directions $\left(\boldsymbol{E}_{\mathrm{L}}, \boldsymbol{E}_{\mathrm{T}}\right)$, shear modulus $\left(\boldsymbol{G}_{\mathrm{LT}}\right)$ and major Poisson's ratio. In this notation, $E_{\mathrm{L}}$ is the stiffness along the interphase in the direction of loading, $\boldsymbol{E}_{\mathrm{T}}$ is the stiffness across the interphase perpendicular to the load and $\boldsymbol{G}_{\mathrm{LT}}$ is the shear stiffness.

Dog-bone tensile specimens, with the interphase either parallel or perpendicular to the direction of loading, were designed to measure $\boldsymbol{E}_{\mathrm{L}}$, and $\boldsymbol{E}_{\mathrm{T}}$, respectively. To measure $G_{\mathrm{LT}}$, a specimen with rectangular shape was designed to mimic a thick-adhered tensilelap specimen [24]. The thick-adhered tensile-lap test was used to measure shear strength and shear modulus of the interphase under a tensile load applied along the interface. Fig. 2 illustrates the geometries of the three specimen types (denoted types I, II and III) used for measuring $\boldsymbol{E}_{\mathrm{L}}, \boldsymbol{E}_{\mathrm{T}}$ and $\boldsymbol{G}_{\mathrm{LT}}$, respectively. For each resin and each of the three specimen designs, seven specimens were tested.

All three types of specimens were tested under quasi-static uniaxial tension. A uniaxial load was applied directly to the type I and II specimens through the ends via serrated-jaw-type end connections. A fixture was designed to hold the type III specimens such that shear stresses were created on the composite by applying a uniaxial tensile load (Fig. 3). The

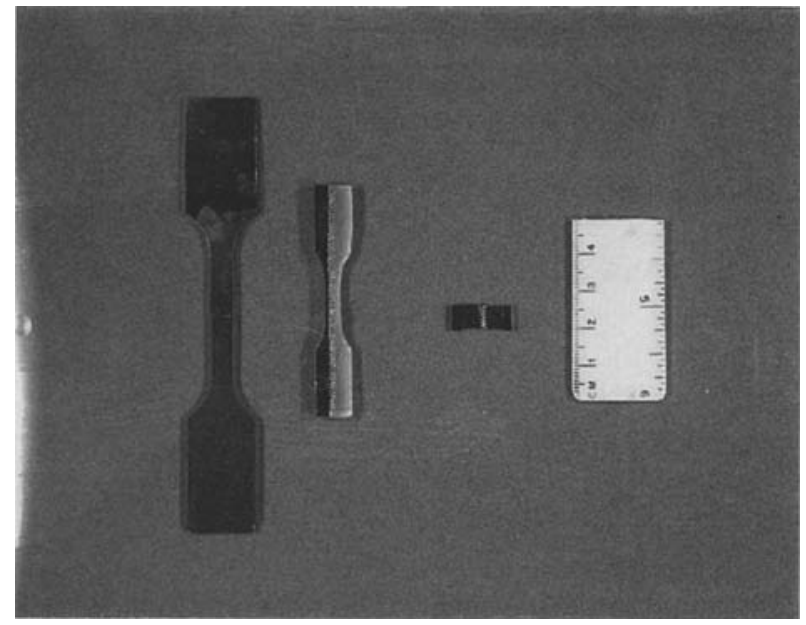

Figure 2 Geometries of specimens used to measure anisotropic elastic constants of bimaterial interphase composites: type I - used to measure longitudinal modulus ( $\left.E_{\mathbf{L}}\right)$; type II - used to measure transverse modulus $\left(E_{\mathbf{T}}\right)$ and type III - used to measure shear $\operatorname{modulus}\left(\boldsymbol{G}_{\mathrm{LT}}\right)$

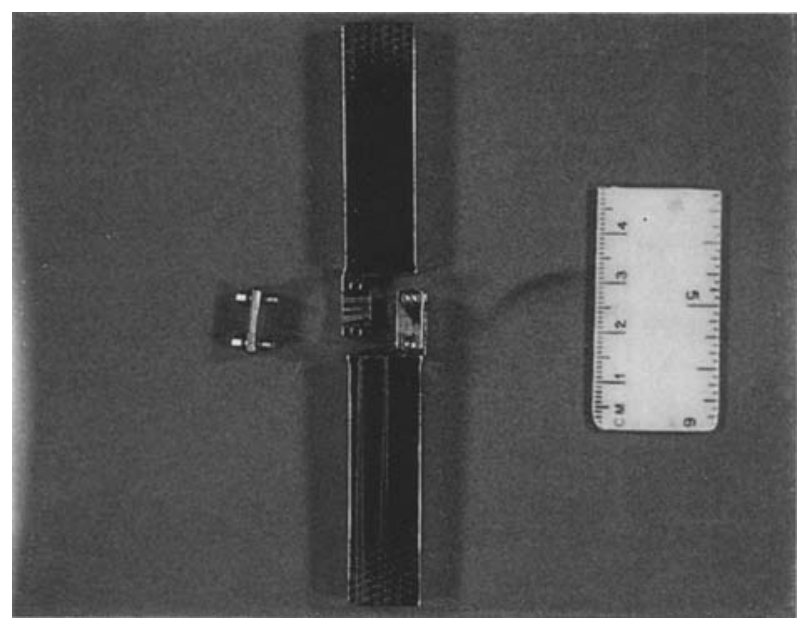

Figure 3 Fixture used to hold shear specimens such that shear stresses were introduced into composite interphase by applying a uniaxial tensile load.

method for measuring the shear modulus was adapted from the ASTM D3983-81 [24].

All specimens were loaded on an Instron 1122 machine at a rate of $0.05 \mathrm{~mm} \mathrm{~min}^{-1}$ until failure. During the tensile tests, the applied load was measured by means of the Instron load cell and the strains parallel to the load were measured by means of an extensometer. Stress-strain curves were plotted and the required material properties were determined. When the applied load was in the longitudinal direction, the initial slope of the stress-strain curve gave the longitudinal modulus $\left(\boldsymbol{E}_{\mathrm{L}}\right)$. Similarly, the transverse $\left(\boldsymbol{E}_{\mathrm{T}}\right)$ and shear moduli $\left(\boldsymbol{G}_{\mathrm{LT}}\right)$ were determined by applying the load in transverse and shear directions, respectively. The mean value of the experimentally measured elastic constant for each type of specimen was compared, using a student's $t$-test, to the value predicted from various composite theories. In other words, the statistical measures tested how close the population means of the measured values were to the predicted values, which were taken as standard or constant values. 


\subsection{Composite theories and analytical methods}

2.3.1. Summary of homogenization theory

Homogenization theory is a mathematical tool developed to analyse the mechanics of microstructured and composite materials $[25,26]$. In this theory, two key assumptions are made. First, the total strain is assumed to be the sum of an apparent strain plus a fluctuating strain:

$$
\varepsilon_{i j}=\bar{\varepsilon}_{i j}+\varepsilon^{*}
$$

In this notation, $\varepsilon_{i j}$ is the total microstructural strain, $\bar{\varepsilon}_{i j}$ is the macroscopic or apparent strain, which varies only on a macroscopic level and $\varepsilon^{*}$ is the fluctuating strain, which varies on both the macroscopic and microscopic levels. The second assumption is that the microstructure displaces periodically and the basic texture can be represented by a unit cell (UC), which is small in comparison to the entire (global) structure. When the global body is loaded, the resulting stresses and strains vary rapidly from point to point because of the small scale of the microstructure relative to the whole structure. In other words, $\varepsilon^{*}$ is periodic on a microstructural scale.

The field variables are described by two material coordinate systems, one macroscopic and one microscopic, which are functions of $x$ and $y$, respectively. The relationship between the two coordinate systems is expressed as:

$$
y=x / \eta
$$

where $\eta$ is the ratio of the dimension of one unit cell to that of the entire structure (i.e. size of local domain relative to global domain). If $\eta$ is small, the total displacement, $\boldsymbol{u}$, on an internal point of the composite is a function of both $x$ and $y$ and is expressed through asymptotic expansion:

$$
\begin{gathered}
u(x, y)=u_{0}(x)+\eta u_{1}(x, y)+\eta^{2} u_{2}(x, y) \\
+\ldots+\eta^{n} u_{n}(x, y)
\end{gathered}
$$

where $u_{0}$ is the macroscopic (global) displacement and all other $u_{i}$ are perturbations in displacements due to the influence of the microstructure.

The expression for $\boldsymbol{u}$ can be incorporated into the weak form of the equilibrium equation:

$$
\int_{\Omega^{\eta}} C_{i j k l} \varepsilon_{i j}(v) \varepsilon_{k l}(u) \mathrm{d} \Omega^{\eta}=\int_{\Gamma} t_{i} v_{i} \mathrm{~d} \Gamma
$$

where $\Omega^{\eta}$ represents the total domain (macroscopic and microscopic) of the composite, $v$ is the virtual displacement and $t_{i}$ are the global tractions. The strains, $\varepsilon$ are obtained by applying the small straindisplacement relationship:

$$
\varepsilon_{i j}(u)=1 / 2\left(u_{i, j}+u_{j, i}\right)
$$

The microscopic and macroscopic equilibrium equations therefore become:

$$
\begin{gathered}
\int_{\Omega^{\eta}} C_{i j k l} \varepsilon_{i j}^{1}(v)\left(\bar{\varepsilon}_{k l}+\varepsilon_{k l}^{*}\right) \mathrm{d} \Omega^{\eta}=0 \\
\int_{\Omega^{\eta}} C_{i j k l} \varepsilon_{i j}^{0}(v)\left(\bar{\varepsilon}_{k l}+\varepsilon_{k l}^{*}\right) \mathrm{d} \Omega^{\eta}=\int_{\Gamma} t_{i} v_{i} \mathrm{~d} \Gamma
\end{gathered}
$$

where: $\varepsilon^{0}$ represents the virtual strains at the macroscopic level and $\varepsilon^{1}$ represents the virtual strains at the microscopic level.

From these relations, two important tensors are determined: (1) the effective elastic constants of the composite, $C_{i j k l}$, and (2) the local structure tensor, $M_{i j k l}$. The effective elastic constants of the interphase account for material inhomogeneity and local geometry. The local structure tensor is a fourth rank tensor which maps global strains into local strains and represents the influence of global strains on the local strain in the media. It can be shown [27] that the local structure tensor is determined through the relationship:

$$
\begin{aligned}
\varepsilon_{i j} & =M_{i j k l} \bar{\varepsilon}_{k l} \\
M_{i j k l} & =1 / 2\left(\delta_{i k} \delta_{j i}+\delta_{i l} \delta_{j k}\right)-\varepsilon_{i j}^{* k l}
\end{aligned}
$$

where $\delta_{i j}$ is the Kronecker delta and $\varepsilon_{i j}^{* k l}$ is the total microstructural strain for the $k l$ th traction. It can also be shown [27] that the apparent stress is the average of the microstress over the unit cell volume and the apparent stiffness, $\bar{C}_{i j k l}$, (apparent stress scaled by apparent strain) is:

$$
\bar{C}_{i j k l}=\mathrm{I} / V_{\text {cell }} \int_{\text {cell }} C_{i j p m} M_{p m k l} \mathrm{~d} V_{\text {cell }}
$$

Equation $6 a$ is solved using a finite element method modified to impose periodic boundary displacements [27]. Equation $6 b$ is solved using standard continuum finite element approaches. The resultant global (macroscopic) strains can be converted into local (microscopic) strains through Equation 7. A flow chart (Fig. 4) shows the finite element modelling regime employed. The local or unit cell model represents the finite element implementation of the microscopic equilibrium Equation $6 a$ and the global model represents implementation of the macroscopic Equation $6 b$. Analysis of the local model under characteristic unit normal and shear volume strains gives the effective interface elastic constants and local structure tensor.

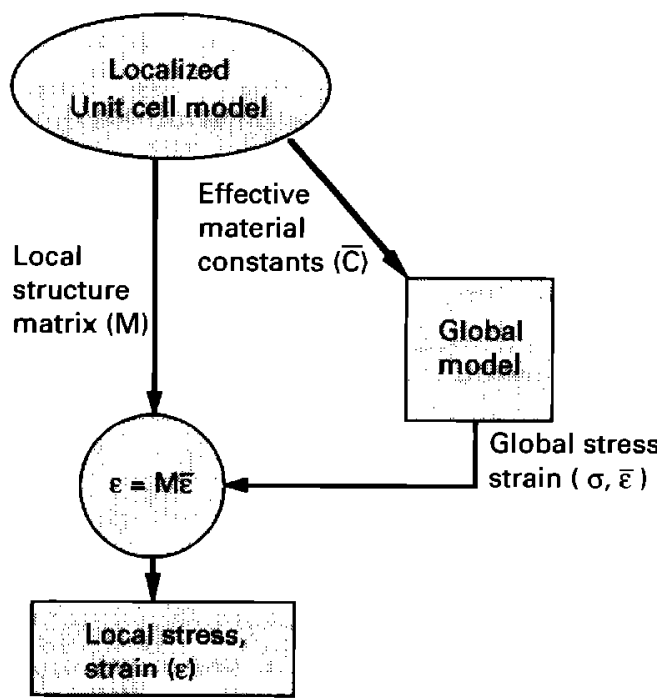

Figure 4 Flowchart outlining homogenization finite element technique 
The global model is solved by inputting the apparent stiffness of the composite $\bar{C}_{i j k l}$, derived from the local model, into the corresponding composite regions of the global model. The resultant global strains are mapped into the local model and local strains are calculated by assembling the local structure tensor. Local stress is determined by relating local strain to material constants at points of interest.

\subsubsection{Homogenization analysis of titanium/resin composite}

A unit cell representative of a repeating microstructure within the interphase of the global composite model was used to calculate the effective elastic (phase), where $X$ is either $E, G, v$ or $K ; V_{i}$ indicates the material volume fraction of the $i$ th phase; and the subscripts 1 and 2 represent titanium and resin, respectively.

The linear rule of mixtures used to calculate material moduli is:

$$
\text { Upper bound: } E_{\mathrm{L}}=E_{1} V_{1}+E_{2} V_{2}
$$

Lower bound: $\boldsymbol{E}_{\mathrm{T}}=E_{1} E_{2} /\left(E_{1} V_{2}+E_{2} V_{1}\right)$

$$
\text { Upper bound: } G_{\mathrm{LT}}=G_{1} V_{1}+G_{2} V_{2}
$$

Lower bound: $G_{\mathrm{LT}}=G_{1} G_{2} /\left(G_{1} V_{2}+G_{2} V_{1}\right)(10 d)$

The Hashin-Shtrikman upper bound is used as the longitudinal modulus of the composite:

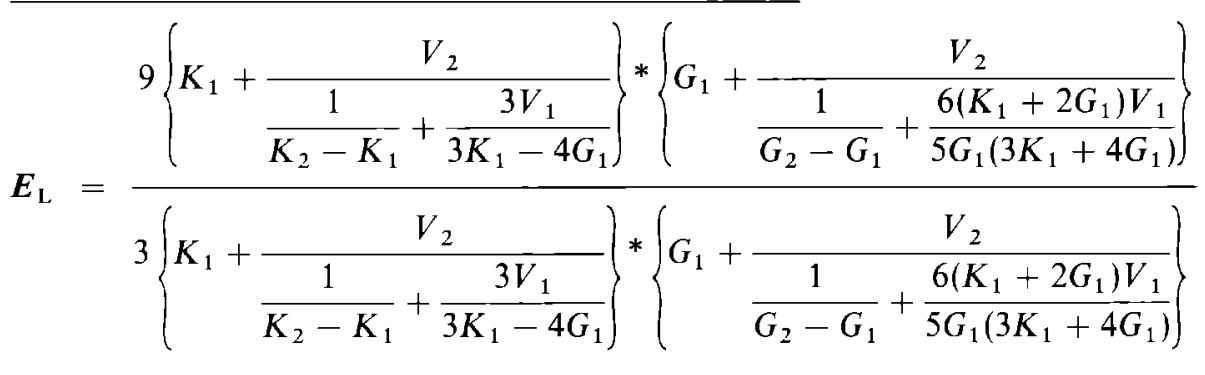

constants of the titanium/resin interphase. The $(900 \times 450 \mu \mathrm{m})$ unit cell contained 550 quadrilateral (Q4) elements and included part of the Ti-6Al-4V substrate, two c.p. titanium microspheres and resin (Fig. 5). The volume fractions of titanium microspheres and resin were the same as in the experimental samples. Each of the materials was assumed to be homogeneous, isotropic and rigidly bonded. The moduli of the individual constituents used in the homogenization analysis were $110 \mathrm{GPa}$ for titanium, $0.2 \mathrm{GPa}$ for epoxy I, and $3 \mathrm{GPa}$ for epoxy II (Table I). The diameter of each microsphere was $1000 \mu \mathrm{m}$. Microspheres contacted one another at two points (nodes). Analysis of the local model yielded the effective interphase elastic constants which were then compared to the experimental data.

\subsubsection{Other composite approaches}

Two standard composite theories were also analysed for their agreement with experimental data and predictions from homogenization theory: the linear rule of mixtures $[11,28,29]$ and Hashin-Shtrikman bounds [12].

Each of these composite laws is based upon the calculation of two of the four elastic constants for two-dimensional isotropy: elastic modulus $(E)$, Poisson's ratio ( $v$ ), shear modulus $(G)$, and bulk modulus $(K)$, with the following relations holding:

$$
\begin{aligned}
G & =E /[2(1+v)] \\
K & =E /[3(1-2 v)]
\end{aligned}
$$

In all of the following relations, $X_{i}$ is the isotropic elastic constant for the $i$ th composite constituent
Interchanging the subscripts 1 and 2 yields the Hashin-Shtrikman lower bound, which is used as the transverse modulus. An upper bound on the Hashin-Shtrikman shear modulus was calculated using Equation 11b. Similarly, interchanging the

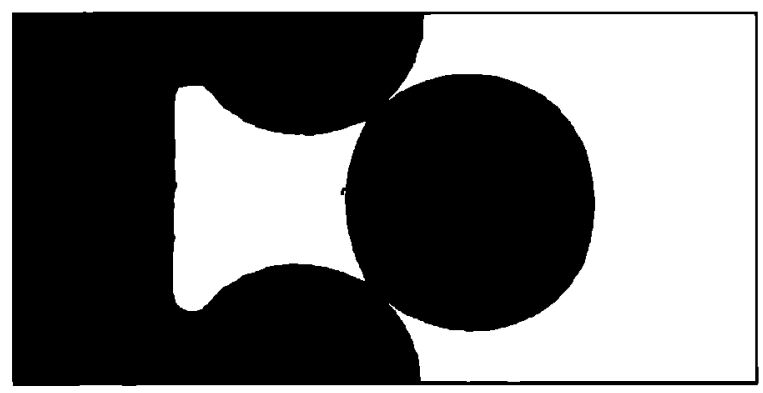

(a)

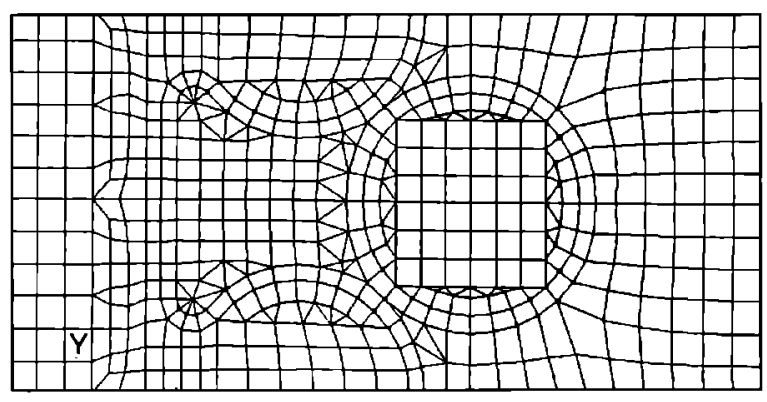

$\mathrm{z} \times$

(b)

Figure 5 (a) Schematic and (b) finite element mesh of local (unit cell) model, which includes edge of $\mathrm{Ti}-6 \mathrm{Al}-4 \mathrm{~V}$ substrate, two commercially pure titanium microspheres and infiltrated resin. 
subscripts yields a lower bound.

$$
G_{\mathrm{LT}}=G_{1}+\frac{V_{2}}{\frac{1}{G_{2}-G_{1}}+\frac{6\left(K_{1}+2 G_{1}\right) V_{1}}{5 G_{1}\left(3 K_{1}+4 G_{1}\right)}}
$$

The lower bounds on the shear modulus were used for comparison with the experimental data since the shear specimens were loaded in the weak direction and this experimental condition most mimics the iso-stress case.

\section{Results}

Table II lists the measured and predicted effective elastic constants. The mean values of the experimentally measured longitudinal moduli were 38.0 and 45.2 GPa for epoxies I and II, respectively. The change in the longitudinal modulus due to epoxy properties was approximately $15 \%$. The experimentally measured transverse moduli were 0.8 and $13.9 \mathrm{GPa}$ and the shear moduli were 0.27 and $2.9 \mathrm{GPa}$ for epoxies I and II, respectively. It is apparent that these latter two effective moduli are much more sensitive to epoxy or "tissue" modulus.

No significant differences (at $p=0.05$ ) in the moduli were found between the experimental measurements and predictions based on homogenization theory, nor between the experimental measurements and Hashin-Shtrikman law. Therefore, the null hypothesis that there is no significant difference in elastic constants between the material moduli measured experimentally and predicted analytically by homogenization theory could not be rejected. For both resins, $\boldsymbol{E}_{\mathrm{L}}$ and $\boldsymbol{E}_{\mathrm{T}}$, measured experimentally, were significantly different from predictions based on the rule of mixtures $(p<0.01)$. Homogenization theory predicted similar results to the Hashin-Shtrikman estimates, but different from the rule of mixtures. In all cases, except for the shear modulus of the composite containing epoxy II, homogenization estimates were within the Hashin-Shtrikman limits, and can therefore be considered reasonable and accurate.

The other null hypothesis addressed was that there is no significant difference between the elastic constants of epoxy I and II specimens. Comparison of the measured moduli for these two groups showed that the differences are significant $(p<0.01)$, rejecting the null hypothesis.

\section{Discussion}

In this study, reproducible measurements of the material properties of a bimaterial interphase composite were developed. There were no statistical differences between experimentally measured effective elastic constants and those predicted with homogenization theory. The statistical measures tested how close the population means of the measured values were to the predicted values, which were taken as standard or constant values. The maximum error in the homogenization technique, determined by comparing homogenization results to standard FEA results, has been determined to be approximately $15 \%$ [23]. The differences between the elastic constants measured experimentally and those predicted by homogenization theory ranged between $4 \%$ and $28 \%$. These differences are possibly attributed to the $15 \%$ differences in results between homogenization and standard FEA. The overall elastic constants obtained from the homogenization model were slightly lower than the experimental measurements. A possible reason for this trend may be that homogenization theory is based upon the assumption that the microstructure is periodic. In the homogenization model, resins are physically dispersed into the porous metal.

The differences between the elastic constants measured experimentally and predicted via homogenization theory were not statistically significant, and differed from metal/tissue interphase properties determined via push-out tests $[30,31]$. Mechanical analyses have also shown that push-out tests do not represent a state of pure interfacial shear and may therefore be inaccurate [32-34].

The results of this study demonstrate that load transfer from an implant surface to the surrounding material is not equivalent in all directions. Load transfer is dominant in the direction parallel to the implant surface because the longitudinal modulus of the interphase is always greater than the transverse modulus. The longitudinal modulus was almost 50 times greater

TA B LE II Experimentally measured versus analytically predicted effective elastic constants

\begin{tabular}{|c|c|c|c|c|c|}
\hline \multirow[b]{2}{*}{ Composite } & \multirow[b]{2}{*}{ Method } & \multicolumn{2}{|c|}{ Axial moduli } & \multicolumn{2}{|c|}{ Shear moduli } \\
\hline & & $E_{\mathrm{T}}(\mathbf{L B})^{\mathrm{a}}$ & $E_{\mathrm{L}}(\mathrm{UB})^{\mathrm{b}}$ & $G_{\mathrm{LT}}(\mathrm{LB})$ & $\boldsymbol{G}_{\mathrm{L}}(\mathrm{UB})$ \\
\hline \multirow[t]{4}{*}{ Ti/epoxy I } & Experimental & $0.80(0.11)$ & $38.0(2.6)$ & $0.27(0.08)$ & - \\
\hline & Homogenization & 0.83 & 34.0 & 0.29 & - \\
\hline & Rule of mixtures & 0.46 & 62.8 & 0.16 & 24.1 \\
\hline & Hashin-Shtrikman & 0.79 & 44.0 & 0.28 & 18.0 \\
\hline \multirow[t]{4}{*}{ Ti/epoxy II } & Experimental & $13.9(3.3)$ & $45.2(4.1)$ & $2.9(1.3)$ & - \\
\hline & Homogenization & 10.6 & 38.5 & 3.7 & - \\
\hline & Rule of mixtures & 7.6 & 64.0 & 2.5 & 24.6 \\
\hline & Hashin-Shtrikman & 10.3 & 46.5 & 3.9 & 18.8 \\
\hline
\end{tabular}

Standard deviations in ()

${ }^{\text {a }} \mathrm{LB}=$ lower bound

${ }^{b} \mathrm{UB}=$ upper bound 
than transverse modulus for the titanium/epoxy I composite and over three times greater for the titanium/epoxy II composite. The longitudinal modulus is determined primarily by the titanium, which is much stiffer than the resins, while the transverse modulus is determined primarily by the softer resin. Both the transverse and shear moduli for the composite containing epoxy II were at least 10 times greater than for the composite with epoxy I. This result implies that the effect of the tissue properties of implant/tissue interphases on load transfer is mainly in the transverse and shear directions.

\subsection{Assumptions and limitations of study}

Tissues grown into porous coatings are heterogeneous and vary in depth, distribution, and percentage [35]. The large number of variable parameters involved in an implant/tissue composite obtained from an in vivo study could reduce the reliability of mechanical testing results [36]. Therefore, in this study resins, which are relatively homogeneous and fully penetrated into the metal pores, were used to eliminate the effect of tissue variability. Homogeneous, isotropic bone tissue can be simulated by using epoxy. The Young's moduli of these resins were within what is considered a reasonable range of properties for healing bone, although it is acknowledged that fully osseointegrated cortical bone will have a substantially higher modulus (e.g. 10-20 GPa). Another reason for using epoxy is that byproducts formed during the curing reactions can be limited. Thus, there was little evidence of voids and material inhomogeneity which may affect experimental measures. The experimental validation of the analytical model is enhanced because an identical system of well-defined constituents was used for both the mechanical testing and the analytical model.

The size of the titanium microspheres $(1000 \mu \mathrm{m})$ was greater than that of commercial porous coating microspheres, which usually range from 200 to $600 \mu \mathrm{m}$. The reason for using $1000 \mu \mathrm{m}$ microspheres was to make the pore size big enough for resin infiltration. However, the composite system used in this study has the same surface metal architecture as a commercial implant, making the results more clinically meaningful.

Currently, only rigid and slip boundary conditions are easily implemented with homogenization FEA codes. Epoxy resins have good adhesive properties to a very broad range of substrates, including titanium. Therefore, the assumption of rigid bonding in the finite element model is valid. This assumption may be more valid for resins than for bone.

Although the experimental measurements were performed on resin/metal composites, this information should also hold true for actual tissue/metal composite measurements, because the material properties of the resins were within the property ranges of actual tissue. However, modifications of the specimens will be required if the methods are to be employed to measure an actual tissue/metal interphase. In order to contain multiple layers of coating particles in the specimen cross-section, the specimens need to be thicker than $1 \mathrm{~mm}$.

\subsection{Comparison to other composite theories}

Classical energy principles are the basic techniques used for determining the bounds on composite moduli. These principles are based upon the premise that the strain energy associated with the actual solution to the field equations is bounded by the strain energy associated with a certain class of "trial" or "admissible" solutions [37]. Estimates of the bounds on composite moduli can be determined from uniform stress or strain boundary conditions or from variational principles. A representative volume element deformed by the application of prescribed surface displacements which yield a uniform strain field provides an upper bound on the overall stiffness tensor. Calculation of the strain energy necessary to satisfy all possible displacement boundary conditions shows that the exact solution is given by the displacement field which minimizes the strain energy [37]. This is known as the principle of minimum potential energy. Likewise, in the principle of minimum complementary energy, a representative volume element loaded by tractions which yield a uniform stress field gives lower bounds on the stiffness.

In a two-phase system, a simplifying assumption is that the stresses and strains in each phase are uniform and the average behaviour of the composite is defined in terms of a representative volume element. The simplest cases for a two-phase composite, assumed to act as rigid inclusions in a rigid matrix, are when the second phase is uniformly aligned either in parallel or in series with an applied uniaxial load. For the case of a parallel arrangement, a uniform strain is assumed in the individual phases, yielding an upper bound on the composite modulus. In the series arrangement, an iso-stress state is assumed, yielding a lower bound. The bounds given by the linear rule of mixtures, sometimes referred to as a Reuss (lower bound) [28] or Voigt (upper bound) [29] model, are generally widely spaced and are often insufficient to model experimental data (e.g. Table II). This implies that iso-strain assumptions for the constituent phases are insufficient. Physically, each phase may be inhomogeneous, individual phase particles may not be physically distinct and may vary in size, shape and orientation. Therefore, on a microstructural level, stresses and strains may not be evenly distributed, and iso-stress or isostrain assumptions are too simplistic.

Improved bounds have been obtained by Hashin and Shtrikman, who took into account the Poisson contraction of the phases [12]. The overall composite response, however, is still assumed to be linear elastic and isotropic. The closeness of the lower and upper bounds is related to the closeness of the constituent moduli $[12,37]$. In general, when the moduli of the phases are close, the Hashin-Shtrikman law can predict values within $10 \%$ of experimental data. In this study, in which the moduli differed by factors of 37 and 550, the Hashin-Shtrikman law still yielded 
results within one-third of the experimental data. For the composite with the more flexible epoxy, only $\boldsymbol{E}_{\mathrm{L}}$ was significantly greater than the experimental data. For the composite with the stiffer epoxy, it was the transverse and shear moduli that deviated more from the experimental data. Predictions based on homogenization theory were closer to experimental data for these cases (Table II). Considering the large differences in phase moduli, the differences between experiment and theory seem quite reasonable. Furthermore, even with greater differences, the HashinShtrikman bounds are useful in testing approximation theories, since solutions outside of the HashinShtrikman bounds are considered invalid. This was a motivation for utilizing these bounds - homogenization estimates were within the Hashin-Shtrikman bounds, further validating the technique.

Through experiments using metal/resin composites, the analytical models predicting composite moduli were validated. Homogenization theory predicts effective elastic properties equivalent to those predicted by the more established Hashin-Shtrikman law. Although the upper and lower bounds of the Hashin-Shtrikman law correspond well to $\boldsymbol{E}_{\mathrm{L}}$ and $\boldsymbol{E}_{\mathrm{T}}$, respectively, it is important to keep in mind that the Hashin-Shtrikman law only predicts properties of isotropic composites. The homogenization method, however, can predict complete anisotropic properties. This study assumed the constituent materials to be isotropic. For the actual case of orthotropic bone, homogenization theory might be more accurate because it can calculate effective moduli for anisotropic constituents. Furthermore, the Hashin-Shtrikman law, when included in a finite element code, can only predict global properties, it cannot estimate local interfacial stresses, which the homogenization method can also predict $[8,23,27]$.

Other variational approaches, models based on composite spheres, composite sphere and cylinder assemblies, elliptical inclusions or laws for fibrereinforced composites, yield solutions close to the Hashin-Shtrikman solutions, or have one bound that collapses into a Hashin-Shtrikman bound $[13,37-41]$. Additionally, such approaches tend to become erroneous for phases with equivalent volume fractions or dissimilar moduli, or for porous media. Having developed reasonable bounds on our data with both the Hashin-Shtrikman and homogenization theories, it was not necessary to pursue alternative composite techniques.

\subsection{Application toward physiological system}

With this experimental validation and an earlier analytical validation [42], we confirm the accuracy of homogenization theory for use in implant/tissue interface mechanics applications. Application of the homogenization technique [23] predicted interfacial elastic constants for a porous titanium/bone interphase as a function of bone stiffness (Table III). For a bone stiffness of $1 \mathrm{GPa}$, the effective stiffness in the direction of loading was $26 \mathrm{GPa}$, whereas the stiffness
TABLE III Effective bone/implant interfacial elastic constants, $C_{i j}$ (GPa) predicted by homogenization theory for different bone properties

\begin{tabular}{lrrrrr}
\hline$C_{\mathrm{ij}}$ & $1 \mathrm{GPa}$ & $5 \mathrm{GPa}$ & $10 \mathrm{GPa}$ & $20 \mathrm{GPa}$ & Orthotropic \\
\hline$E_{\mathrm{L}}$ & 25.70 & 30.10 & 35.30 & 44.90 & 44.70 \\
$E_{\mathbf{T}}$ & 2.30 & 10.00 & 18.30 & 32.60 & 21.20 \\
$G_{\mathbf{L T}}$ & 0.80 & 3.60 & 6.70 & 12.30 & 9.40 \\
$v_{\mathbf{L T}}$ & 0.29 & 0.30 & 0.30 & 0.30 & 0.30 \\
$v_{\mathbf{T L}}$ & 0.03 & 0.10 & 0.16 & 0.22 & 0.25 \\
\hline
\end{tabular}

across the interphase and the shear stiffness were an order of magnitude less, only $2 \mathrm{GPa}$ and $0.8 \mathrm{GPa}$, respectivaly. The composite interface is therefore more compliant in the transverse direction. These trends are similar to those predicted and experimentally validated with resins. When bone stiffness is increased, the absolute values of the effective elastic constants increase, but the relative values between the different elastic constants remain the same. Even though the elastic constants of the constituent materials were isotropic, the effective elastic constants indicate that the interfacial zone is orthotropic. Since the interphase is anisotropic, with stiffness an order of magnitude lower across the interphase, local mechanics, tissue ingrowth and remodelling may be directionally dependent.

Porous coated implants interface with a substantial amount of cortical bone, which has a higher modulus than the epoxies used in this study. Since a linear theory of homogenization was used and tissue was assumed to be homogeneous, the trends in predicted effective moduli will not change with increasing bone moduli, only the absolute values will change. In a study which parametrically varied bone modulus, the magnitude of the local stresses and strains varied with bone modulus, but the distribution remained the same $[23,43]$. Second, most composite theories offer better agreement with experimental data if differences in material moduli are minimized. Using moduli more representative of cortical bone would therefore offer better agreement between analytical predictions and experimental data, providing a stronger validation of homogenization theory.

It is well known that bone can adapt to stresses and strains. The degree of osseointegration into a cementless implant is a possible result from such adaptation. Our general hypothesis is that the local material and tissue architecture within the interphase is the major factor determining the directions of load transfer, and therefore regulates osseointegration and adaptation. This study provides a first step towards quantifying interphase elasticity and the local mechanical environment of the interphase. Further quantification of local stresses and strains in implant/tissue interphases and testing of our hypothesis may aid improvements in implant design.

\section{Conclusions}

(1) Homogenization theory predicts the same effective elastic constants for a bimaterial interphase composite as those measured experimentally. 
(2) Homogenization theory is as accurate as the Hashin-Shtrikman theory, is also valid when individual phases are anisotropic, and can determine local interphase stresses and strains.

(3) The use of these effective elastic constants in the interphase of a global finite element model is therefore reliable.

(4) The computational predictions made with homogenization theory also reflect the influence of each constituent on the resultant mechanical properties of the composite. Thus the use of homogenization theory to estimate local properties of an implant/tissue interphase becomes feasible even though the tissue is heterogeneous.

(5) Such analyses can also be used to determine the effective elastic constants of fibre/matrix or particulate composites with accuracy.

\section{Acknowledgements}

This work was supported, in part, by NIH 2-SO7RR05321, 5-P60-AR20557 and R01-AR34399.

\section{References}

I. L. D. DORR, M. LUCKETT and J. P. CONATY, Clin. Orthop. 260 (1990) 215.

2. J. D. BOBYN, R. M. PILliAR, H. U. CAMERON and G. C. WEATHER L Y, ibid. 150 (1980) 263.

3. S. YUE, R. M. PILliAR and G. C. WEATHERLY, J. Biomed. Mater. Res. 18 (1984) 1043.

4. S. D. COOK, F. S. GEORGETTE, H. B. SKINNER and R. J. HA DDAD Jr., ibid. 18 (1984) 497.

5. D. H. KOHN and P. DUCHEYNE, ibid. 24 (1990) 1483

6. D. WOLFARTH, M. FILIAGGI and P. DUCHEYNE, J. Appl. Biomater. 1 (1990) 3.

7. D. R. CARTER and N. J. GIORI, in "The bone biomaterial interface", edited by J. E. Davies (University of Toronto Press, Toronto, 1991) p. 367.

8. C. C. K O, PhD dissertation, University of Michigan (1994).

9. C. C. KO, D. H. KOHN, S. J. HOLLISTER and S. A. GOLDSTEIN, in Trans. Soc. for Biomater. 21 (1995) 318.

10. R. HILL, J. Mech. Phys. Solids 11 (1963) 375.

11. B. PAUL Trans. Amer. Inst. Mech. Eng. 36 (1960) 218.

12. Z. HASHIN and S. SHTRIKMAN, J. Mech. Phys. Solids $\mathbf{1 1}$ (1963) 127.

13. Z. HASHIN, J. Appl. Mech. $\mathbf{5 0}$ (1983) 481

14. D. D. MOYLE, J. J. KLAWITTER and S. F. HULBERT, J. Biomed. Mater. Res. S vmp. 4 (1973) 363.

15. P. DUCHEYNE, E. AERNOUDT, P. DE MEESTER, M. MARTENS J. C. MULIER and D. VAN LEEUWEN. J. Biomech. 11 (1978) 297.
16. A. ROHLMANN, E. J. ChEAL, W. C. HAYES and G. BERGMANN, ibid. 21 (1988) 605.

17. A. SHIR AZI-ADL and A. M. AHMED, Ann. Biomed. Eng. 17 (1989) 411.

18. T. M. KEAVENY and D. L. BARTEL. J. Biomech. 27 (1994) 1147.

19. D. R. PEDERSON, T. D. BROWN and R. A. BRAND, ibid. 24 (1991) 1131.

20. P. B. MESSER SMITH and F. W. COOK E. J. Biomed. Mater. Res. 24 (1990) 591.

21. C. C. KO, D. H. KOHN and S. J. HOLliSTER, J. Oral Implantol. 18 (1992) 220.

22. Idem., Trans. Orthop. Res. Soc. 17 (1992) 74.

23. D. H. KOHN, C. C. KO and S. J. HOLLISTER, in ASME BED-Vol. 24 "Bioengineering Conference", edited by N. A. Langrana (Amer. Soc. of Mech. Eng., New York, 1993) p. 335.

24. ASTM Standard D3983-81, ASTM, Philadelphia, PA, 1991.

25. E. SANCHEZ-PALENCIA, in "Non-homogeneous media and vibration theory" (Springer, Berlin, 1980).

26. P. M. SUQUET, in "Homogenization techniques for composite media", edited by E. Sanchez-Palencia and A. Zaoui (Springer, Berlin, 1985).

27. S. J. HOLLISTER, D. P. FYHRIE, K. J. JEPSEN and S. A. GOLDSTEIN, J. Biomech. 24 (1991) 825.

28. W. VOIGT, "Lehrbuch der Kristallphysik" (Teubner, Berlin, 1910।.

29. A. REUSS, Zeitschrift fur Angewandte Mathematik und Mechanik 9 (1929) 49

30. A. J. T. CLEMOW, A. M. WEINSTEIN, J. J. KLAWITTER, J. KOENEMAN and J. ANDER SON, J. Biomed. Mater. Res. 15 (1981) 73.

31. R. C. ANDERSON, S. D. COOK. A. M. WEINSTEIN and R. J. HADDAD, Jr., Clin. Orthop. 182 (1984) 242.

32. A. SHIR AZI-A DL. J. Biomech. Eng. 114 (1992) 111

33. Idem., ibid. 114 (1992) 155.

34. W. J. A. DHERT. C. C. P. M. VERHEYEN, L. H. BRAAK, J. R. DE WIJN. C. P. A. T. KLEIN, K. DE GROOT and P. M. ROZING, J. Biomed. Mater. Res. 26 (1992) 119.

35. S. D. COOK. K. A. THOMAS and R. J. HADDAD, Jr. Clin. Orthop. 234 (1988) 90.

36. J. BLACK, J. Biomed. Mater. Res. 23 (1989) 1243.

37. N. LAWS, in "Physics of modern materials", Vol. I (International Atomic Energy Agency, Vienna, 1980) p. 465.

38. S. AHMED and F. R. JONES, J. Mater. Sci. 25 (1990) 4933.

39. J. D. ESHELB Y. Proc. Roy. Soc. Lond. A241 (1957) 376.

40. R. HIL L, J. Mech. Phys. Solids 12 (1964) 199.

41. Z. HASHIN and B. W. ROSEN, J. Appl. Mech. 31 (1964) 223.

42. D. H. KOHN, C. C. KO and S. J. HOLLISTER, in Trans. Orthop. Res. Soc. 18 (1993) 469.

43. D. H. KOHN, C. C. KO and S. J. HOLLISTER, in ASME BED-Vol. 26 "Advances in Bioengineering 1993", edited by J. M. Tarbell (Amer. Soc. of Mech. Eng.., New York, 1993) p. 231.

Received 6 March

and accepted 6 June 1995 